Article

\title{
Serum Exosomal miRNAs for Grading Hepatic Fibrosis Due to Schistosomiasis
}

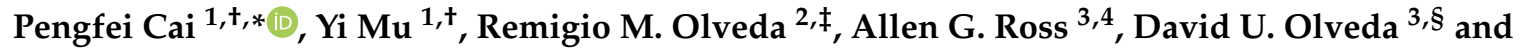 \\ Donald P. McManus ${ }^{1, *}$ \\ 1 Molecular Parasitology Laboratory, QIMR Berghofer Medical Research Institute, Brisbane 4006, Australia; \\ Yi.Mu@qimrberghofer.edu.au \\ 2 Department of Health, Research Institute for Tropical Medicine, Manila 1781, Philippines; \\ rolvedamd_ritm_doh@yahoo.com \\ 3 Menzies Health Institute Queensland, Griffith University, Gold Coast 4222, Australia; \\ a.ross@griffith.edu.au (A.G.R.); dvdolveda@gmail.com (D.U.O.) \\ 4 International Centre for Diarrhoeal Disease Research, Bangladesh (ICDDR, B), Dhaka 1212, Bangladesh \\ * Correspondence: Pengfei.Cai@qimrberghofer.edu.au (P.C.); Don.McManus@qimrberghofer.edu.au (D.P.M.); \\ Tel.: +61-7-3362-0406 (P.C.); +61-7-3362-0401 (D.P.M.) \\ + These two authors contributed equally to this work. \\ $\ddagger$ Deceased April 24th, 2020. \\ $\S$ Current address: Department of Pathology, JONELTA Foundation School of Medicine, \\ University of Perpetual Help Rizal, Manila, Philippines.
}

Received: 15 April 2020; Accepted: 14 May 2020; Published: 18 May 2020

\begin{abstract}
Chronic infection with Schistosoma japonicum or Schistosoma mansoni results in hepatic fibrosis of the human host. The staging of fibrosis is crucial for prognosis and to determine the need for treatment of patients with schistosomiasis. This study aimed to determine whether there is a correlation between the levels of serum exosomal micro-ribonucleic acids (miRNAs) (exomiRs) and fibrosis progression in schistosomiasis. Reference gene (RG) validation was initially carried out for the analysis of serum exomiRs expression in staging liver fibrosis caused by schistosome infection. The expression levels of liver fibrosis-associated exomiRs in serum were determined in a murine schistosomiasis model and in a cohort of Filipino schistosomiasis japonica patients $(n=104)$ with different liver fibrosis grades. Of twelve RG candidates validated, miR-103a-3p and miR-425-5p were determined to be the most stable genes in the murine schistosomiasis model and subjects from the schistosomiasis-endemic area, respectively. The temporal expression profiles of nine fibrosis-associated serum exomiRs, as well as their correlations with the liver pathologies, were determined in C57BL/6 mice during $S$. japonicum infection. The serum levels of three exomiRs (miR-92a-3p, miR-146a-5p and miR-532-5p) were able to distinguish subjects with fibrosis grades I-III from those with no fibrosis, but only the serum level of exosomal miR-146a-5p showed potential for distinguishing patients with mild (grades 0-I) versus severe fibrosis (grades II-III). The current data imply that serum exomiRs can be a supplementary tool for grading liver fibrosis in hepatosplenic schistosomiasis with moderate accuracy.
\end{abstract}

Keywords: schistosomiasis; Schistosoma japonicum; hepatic fibrosis; exosomal microRNAs; exomiRs; biomarker

\section{Introduction}

Schistosomiasis, a major public health problem, affects 230 million people worldwide [1,2]. Three species of schistosomes (trematode blood flukes), Schistosoma haematobium, S. mansoni, and S. japonicum, are the most clinically relevant. The infection of the latter two species results in the development of 
hepatopathology which is trigged by soluble egg antigen (SEA) released by eggs lodged in liver tissue. Several different types of immune cells (eosinophils, neutrophils, macrophages, and lymphocytes) are recruited to the periphery of deposited eggs and, along with resident liver cells, participate in the process [3,4]. Accumulating evidence shows that specific micro-ribonucleic acids (miRNAs) are dysregulated in particular cell types within the liver tissue, as well as in the circulatory system, during the development of liver fibrosis [5-9].

The gold standard for the diagnosis of liver fibrosis involves liver biopsy, which is highly invasive. In addition, serological diagnosis of liver fibrosis is currently challenging, due to the existing clinical biomarkers that have narrow applicability due to their lack of specificity and sensitivity [10]. The discovery of extracellular vesicles (EVs), small membranous nano-vesicles secreted by a variety of cell types, has evoked considerable interest for biomarker discovery, particularly in the field of cancer [11-13]. EVs include three main types; exosomes, microvesicles, and apoptotic bodies, based on their composition, biogenesis, and size [14]. EVs can transmit a wealth of bioactive cargos, such as DNA, messenger RNAs (mRNAs), miRNAs, proteins, and lipids, between cells, thus playing a vital role in cell-cell communication [15]. EVs stably circulate in many kinds of body fluids and protect the packaged nucleic acids from degradation. Serum/plasma EVs miRNAs also have been recently tested as being prospective predictors of virus-related hepatic fibrosis [7,8]. These two studies evaluated whether EV-derived miRNA signatures can provide a superior diagnostic performance than their counterparts isolated directly from serum/plasma in predicting the severity of liver fibrosis, yet contradictory results were obtained $[7,8]$. We recently measured the levels of circulating miRNAs directly isolated from serum to trace the progression of hepatic fibrosis during an infection caused by S. japonicum [9]. These investigations provide a key first step in identifying unique circulating host miRNAs associated with schistosome-associated liver fibrosis. We then considered it would be informative to determine whether measurement of serum exosomal miRNAs (exomiRs) can improve the ability to grade fibrosis in schistosomiasis. In this study, the widely used term 'exosomes' was used to refer to EVs, in general, unless otherwise specified.

Real-Time Quantitative Reverse Transcription PCR (qRT-PCR) is a powerful and widely used method to detect the levels of miRNAs in a variety of cell lines, tissues, and body fluids [16]. One prerequisite for valid analysis of qRT-PCR data is the normalization to the most stably expressed endogenous reference genes (RGs), particularly for assessment of gene expression in extracellular vesicles. To date, no reliable RGs have been identified that can be used to assess the levels of exosomal target miRNAs in serum/plasma during the development of liver fibrosis.

In light of our previous encouraging results [9], we report on further validation of serum exomiRs to improve on liver fibrosis grading in a mouse model of schistosomiasis and then in humans. C57BL/6 mice were initially employed to identify endogenous RGs in order to analyze serum exomiRs, and to determine the temporal expression profiles of nine exomiR candidates in serum using qRT-PCR assays during $S$. japonicum infection; the association between the expression levels of the investigated exomiRs with the severity of liver pathology was then determined. Then, the most stable endogenous RGs were identified within 12 exomiR candidates in a subset of cohort of individuals resident in a rural schistosomiasis-endemic area of the Philippines. Finally, 11 selected exomiR candidates were validated within serum samples from a cohort of schistosomiasis japonica patients with different grades of liver fibrosis. The study has further identified serum exomiRs that could serve as biomarkers for assessing the progression of hepatic fibrosis due to schistosomiasis in a rural endemic setting.

\section{Results}

\subsection{Identification of Endogenous RGs for Serum exomiR Expression Analysis in C57BL/6 Mice During} Schistosome Infection

To accurately quantify exosome derived miRNAs, the identification of appropriate endogenous reference genes (RGs) as calibrator is key. Based on the literature, 12 previously reported RGs tested in 
plasma/serum/cell extracellular vesicles/exosomes (Table 1) were selected for validation in a murine schistosomiasis model initially.

Table 1. Selected endogenous reference micro-ribonucleic acid (miRNA) candidates.

\begin{tabular}{cccc}
\hline miRNA Name & Accession Number & Sequence & References \\
\hline let-7a-5p & MIMAT0000062 & UGAGGUAGUAGGUUGUAUAGUU & {$[17,18]$} \\
miR-16-5p & MIMAT0000069 & UAGCAGCACGUAAAUAUUGGCG & {$[19]$} \\
miR-23a-3p & MIMAT0000078 & AUCACAUUGCCAGGGAUUUCC & {$[19,20]$} \\
miR-26a-5p & MIMAT0000082 & UUCAAGUAAUCCAGGAUAGGCU & {$[17,18,20]$} \\
miR-101-3p & MIMAT0000099 & UACAGUACUGUGAUAACUGAA & {$[20]$} \\
miR-103a-3p & MIMAT0000101 & AGCAGCAUUGUACAGGGCUAUGA & {$[17]$} \\
miR-221-3p & MIMAT0000278 & AGCUACAUUGUCUGCUGGGUUUC & {$[17,18]$} \\
miR-423-5p & MIMAT0004748 & UGAGGGGCAGAGAGCGAGACUUU & {$[21]$} \\
miR-425-5p & MIMAT0003393 & AAUGACACGAUCACUCCCGUUGA & {$[21]$} \\
miR-181a-5p & MIMAT0000256 & AACAUUCAACGCUGUCGGUGAGU & {$[18]$} \\
miR-191-5p & MIMAT0000440 & CAACGGAAUCCCAAAAGCAGCUG & {$[18]$} \\
miR-631 & MIMAT0003300 & AGACCUGGCCCAGACCUCAGC & {$[22]$} \\
\hline
\end{tabular}

After quantification of the RG candidates in serum exosome samples extracted from C57BL/6 mice during the infection course by RT-PCR, gene expression stability was analyzed using NormFinder, geNorm, BestKeeper, and Delta Ct to rank the RGs (Figure 1). NormFinder, BestKeeper, and Delta Ct identified miR-103a-3p as the most stable candidate, while geNorm identified both miR-103a-3p and miR-221-3p as the most stable candidates (Figure 1a-d). All algorithms identified let-7a-5p as the most unstable candidate. The geNorm ranked miR-103a-3p (0.305), miR-221-3p (0.305), and miR-191-5p (0.324) as being among the most stable RGs (Figure 1b). Using BestKeeper analysis, miR-103a-3p (0.471), miR-221-3p (0.509), and miR-191-5p (0.533) showed the lowest variation (Figure 1c). The geometric mean of all four above-mentioned methods was used to yield the final list of RG candidates, which identified miR-103a-3p (1.0), miR-221-3p (2.21) and miR-181a-5p (3.16) as being among the most stable genes, with miR-23a-3p, miR-16-5p, and let-7a-5p being the least stable species (Figure 1e).

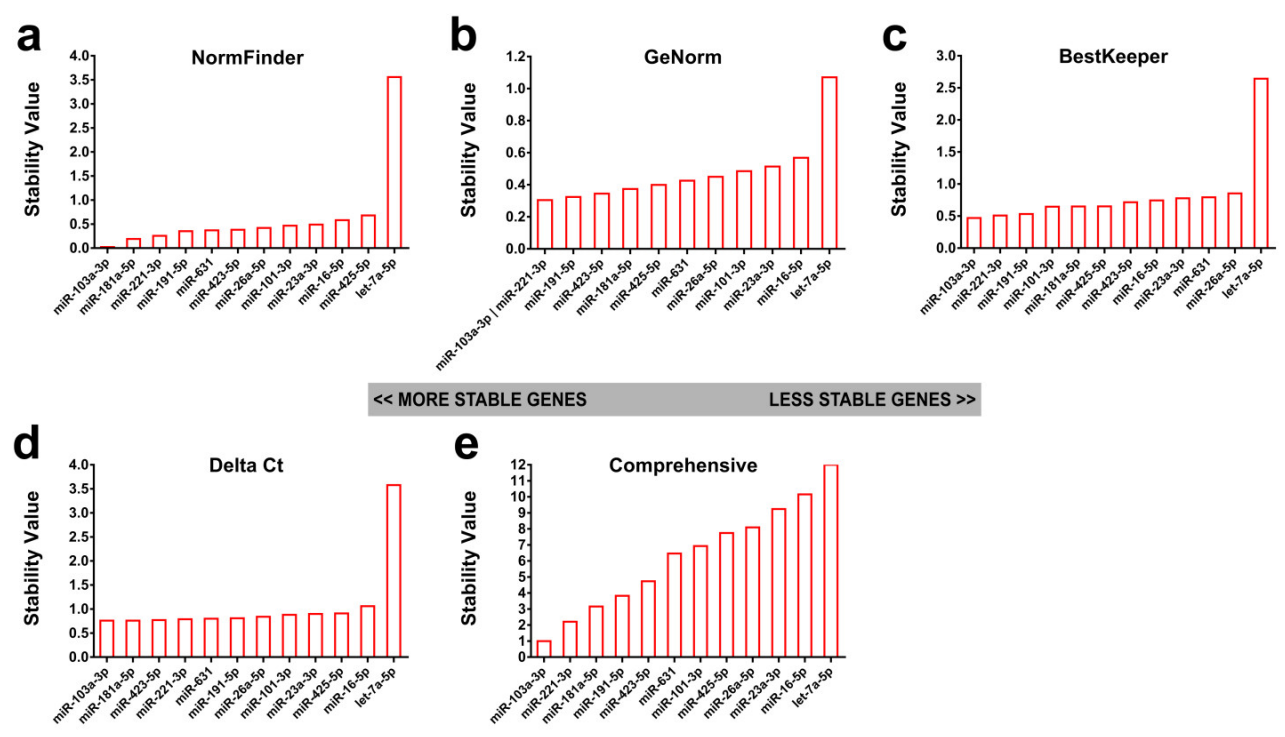

Figure 1. Identification of candidate reference genes (RGs) from Real-Time Quantitative Reverse Transcription PCR (qRT-PCR) quantification data of 12 exosomal microRNAs (exomiRs) in forty serum samples collected from C57BL/6 mice across the $S$. japonicum infection course $(0$ week p.i., $n=7 ; 4$ weeks p.i., $n=7 ; 6$ weeks p.i., $n=6 ; 7$ weeks p.i., $n=6 ; 9$ weeks p.i., $n=7 ; 11$ weeks p.i., $n=7)$. Data were analyzed using (a) NormFinder, (b) GeNorm, (c) BestKeeper, and (d) Delta Ct. (e) A comprehensive ranking of candidate RGs was produced by RefFinder by integrating results of the above four algorithms. 


\subsection{Temporal Expression of Serum Exosomal miRNAs in C57BL/6 Mice During the Course of S. japonicum} Infection

Based on previous studies [7-9,23,24], nine fibrosis-associated miRNA candidates were selected to test if their levels in serum exosomes were capable of predicting the grades of liver fibrosis during schistosomiasis progression (Table 2). Temporal expression of the nine exomiRs in serum was initially explored in C57BL/6 mice post-S. japonicum-infection with the most stable, exomiR miR-103a-3p, identified earlier used as an endogenous RG (Figure 2). Five of the nine exomiRs (miR-150-5p, let-7a-5p, let-7d-5p, miR-200b-3p, and miR-146a-5p) were significantly down-regulated during the infection course; the levels of three exomiRs, miR-150-5p, miR-200b-3p, and let-7a-5p, in serum were significantly down-regulated from 4 week p.i. and onwards, while the serum level of exomiR miR-146a-5p was also significantly down-regulated from 4 week p.i. and thereafter, except at 9 weeks p.i. Decreased expression in the level of serum exomiR let-7d-5p was observed from 6 weeks p.i. and subsequently. For the other exomiRs tested, the levels of miR-92a-3p and miR-151-3p were down-regulated only at 6 weeks p.i. The level of miR-532-5p in serum exosome was found significantly reduced at 4 and 6 weeks p.i., yet was up-regulated at 9 weeks p.i. No statistically significant alternation in the serum level of exosomal miR-192-5p was observed over the infection course.

Table 2. Candidate miRNAs for grading liver fibrosis based on available literature.

\begin{tabular}{cccc}
\hline miRNA & Accession Number & Sequence & References \\
\hline miR-92a-3p & MIMAT0000092 & UAUUGCACUUGUCCCGGCCUGU & {$[8,9]$} \\
miR-150-5p & MIMAT0000451 & UCUCCCAACCCUUGUACCAGUG & {$[8,9]$} \\
miR-192-5p & MIMAT0000222 & CUGACCUAUGAAUUGACAGCC & {$[8,9]$} \\
miR-200b-3p & MIMAT0000318 & UAAUACUGCCUGGUAAUGAUGA & {$[8,9]$} \\
let-7a-5p & MIMAT0000062 & UGAGGUAGUAGGUUGUAUAGUU & {$[7,9,23]$} \\
let-7d-5p & MIMAT0000065 & AGAGGUAGUAGGUUGCAUAGUU & {$[7,9]$} \\
miR-146a-5p & MIMAT0000449 & UGAGAACUGAAUUCCAUGGGUU & {$[9]$} \\
miR-151-3p & MIMAT0000757 & CUAGACUGAAGCUCCUUGAGG & {$[24]$} \\
miR-532-5p & MIMAT0002888 & CAUGCCUUGAGUGUAGGACCGU & {$[24]$} \\
\hline
\end{tabular}

2.3. Correlations of the Serum Levels of exomiRs with Liver Pathological Parameters in C57BL/6 Mice during S. japonicum Infection

We then determined the correlations between the serum levels of the nine exomiRs and hepatic pathological parameters in C57BL/6 mice during the infection course (Table 3 and Figure 3). The levels of two exomiRs miR-151-3p and miR-192-5p showed a significant positive correlation with the serum levels of alanine aminotransferase (ALT) and aspartate aminotransferase (AST), while the levels of five exomiRs (miR-92a-3p, miR-150-5p, miR-200b-3p, let-7a-5p, and let-7d-5p) displayed a significant inverse correlation with the serum levels of liver enzymes (Table 3). The levels of two exomiRs (miR-192-5p and miR-532-5p) positively correlated with liver HP content, the intensity of liver fibrosis and the degree of granulomatous involvement in the liver; while the other two exomiRs (miR-200b-3p and let-7d-5p) negatively correlated with these liver pathological parameters (for miR-192-5p, $r=0.4253$, 0.3850 , and 0.3524 , and $p=0.0062,0.0141$, and 0.0257, respectively; for miR-532-5p, $r=0.7162,0.6004$, and 0.5760 , and $p<0.0001,<0.0001$, and $=0.0001$, respectively; for miR-200b-3p, $r=-0.4392,-0.5915$, and -0.6210 , and $p=0.0046,<0.0001$, and $<0.0001$, respectively; for let-7d-5p, $r=-0.3728,-0.5629$, and -0.6063 , and $p=0.0178,=0.0002$, and $<0.0001$, respectively). The level of serum exosomal let-7a-5p significantly correlated with the intensity of hepatic fibrosis, the degree of granulomatous involvement and necrosis formed in the liver $(r=-0.4939,-0.5529$, and -0.3338 , and $p=0.0012,0.0002$, and 0.0353 , respectively). For the levels of miR-92a-3p and miR-146a-5p, the significant correlations were only observed with the level of liver necrosis $(r=-0.3664$ and -0.3625 , and $p=0.0201$ and 0.0215 , respectively). The serum levels of another three exomiRs, miR-150-5p, miR-200b-3p, and miR-151-3p, also correlated with the intensity of liver necrosis in C57BL/6 mice during S. japonicum infection. 

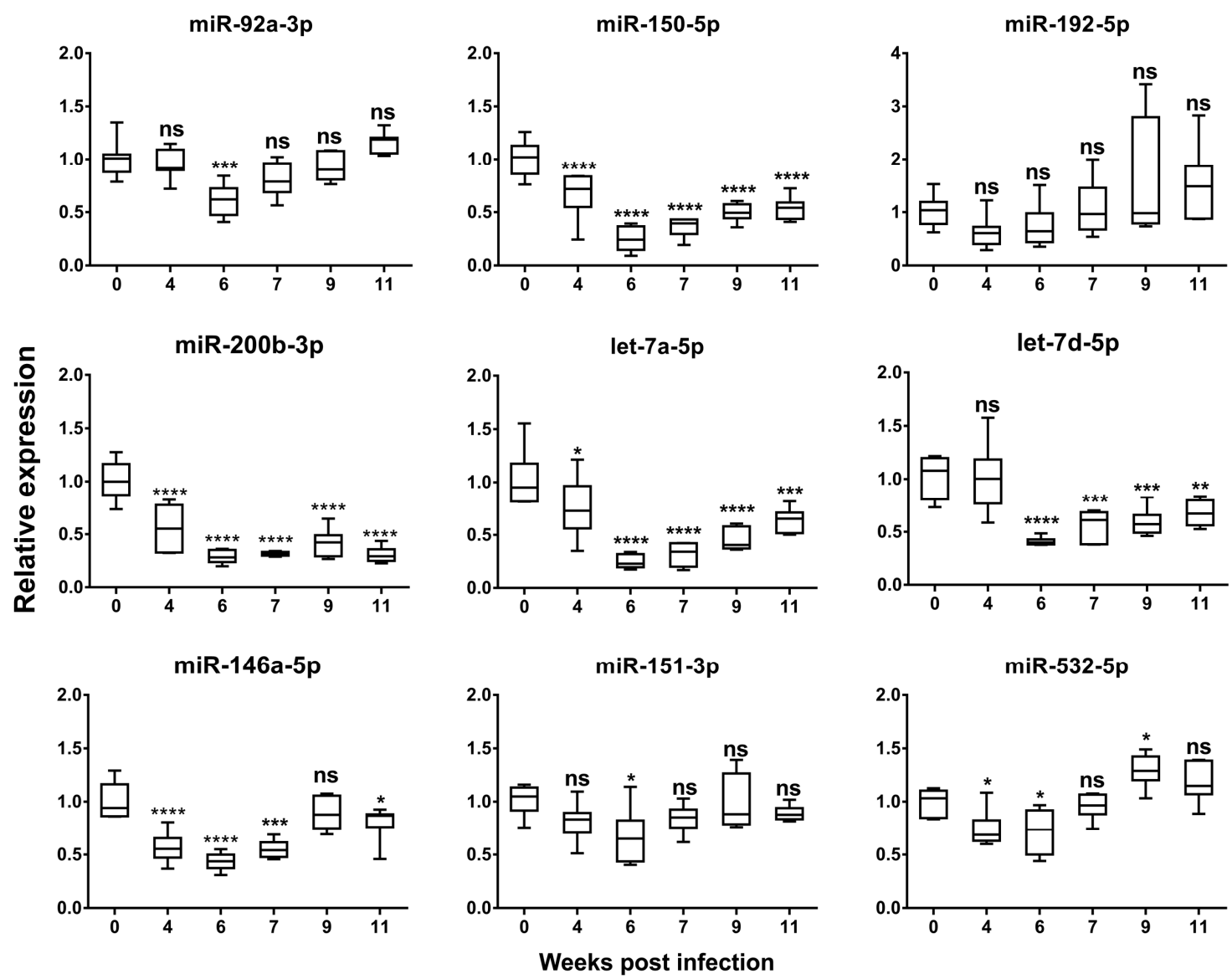

Figure 2. Temporal expression of serum exomiRs in C57BL/6 mice during $S$. japonicum infection. (Week 0, $n=7$; week 4,n=7; week 6, n=6; week 7, $n=6$; week 9, $n=7$; week 11, n= 7). Boxes represent the interquartile range of the data. The hash marks above and below the boxes indicate the 90th and 10th percentiles for each group, respectively, while the lines across the boxes indicate the median values. $p$ values were calculated using One-way ANOVA. (ns = no significant difference, $\left.{ }^{*}=p<0.05,{ }^{* *}=p<0.01,{ }^{* * *}=p<0.001,{ }^{* * * *}=p<0.0001\right)$.

Table 3. Correlations of the levels of exosomal miRNAs (exomiRs) in serum with liver pathological parameters in C57BL/6 mice during S. japonicum infection.

\begin{tabular}{|c|c|c|c|c|c|c|c|c|c|c|c|c|}
\hline \multirow{2}{*}{ exomiR } & \multicolumn{2}{|c|}{ AST } & \multicolumn{2}{|c|}{ ALT } & \multicolumn{2}{|c|}{ HP } & \multicolumn{2}{|c|}{$\mathbf{H F}^{\#}$} & \multicolumn{2}{|c|}{ GRA $^{\#}$} & \multicolumn{2}{|c|}{ NEC $^{\#}$} \\
\hline & $r$ & $p$ & $r$ & $p$ & $r$ & $p$ & $r$ & $p$ & $r$ & $p$ & $r$ & $p$ \\
\hline miR-92a-3p & -0.4716 & 0.0021 & -0.4126 & 0.0082 & 0.2405 & 0.1350 & 0.1310 & 0.4205 & 0.0695 & 0.6700 & -0.3664 & 0.0201 \\
\hline miR-150-5p & -0.5213 & 0.0006 & -0.6346 & $<0.0001$ & -0.2985 & 0.0614 & -0.4820 & 0.0016 & -0.5347 & 0.0004 & -0.4191 & 0.0071 \\
\hline miR-192-5p & 0.4035 & 0.0098 & 0.5393 & 0.0003 & 0.4253 & 0.0062 & 0.3850 & 0.0141 & 0.3524 & 0.0257 & -0.0333 & 0.8386 \\
\hline miR-200b-3p & -0.4081 & 0.0089 & -0.5874 & $<0.0001$ & -0.4392 & 0.0046 & -0.5915 & $<0.0001$ & -0.6210 & $<0.0001$ & -0.3402 & 0.0317 \\
\hline let-7a-5p & -0.4960 & 0.0011 & -0.5719 & 0.0001 & -0.2869 & 0.0727 & -0.4939 & 0.0012 & -0.5529 & 0.0002 & -0.3338 & 0.0353 \\
\hline let-7d-5p & -0.5382 & 0.0003 & -0.6207 & $<0.0001$ & -0.3728 & 0.0178 & -0.5629 & 0.0002 & -0.6063 & $<0.0001$ & -0.2523 & 0.1163 \\
\hline miR-146a-5p & -0.2083 & 0.1972 & -0.2196 & 0.1734 & 0.2567 & 0.1098 & 0.0116 & 0.9435 & -0.0413 & 0.8002 & -0.3625 & 0.0215 \\
\hline miR-151-3p & 0.5395 & 0.0003 & 0.5304 & 0.0004 & -0.1533 & 0.3451 & 0.0869 & 0.5941 & 0.1647 & 0.3099 & 0.3702 & 0.0187 \\
\hline miR-532-5p & -0.1504 & 0.3544 & -0.1137 & 0.4849 & 0.7162 & $<0.0001$ & 0.6004 & $<0.0001$ & 0.5760 & 0.0001 & -0.2977 & 0.0621 \\
\hline
\end{tabular}

Correlations were analyzed by Pearson's $\mathrm{r}(n=40)$. Abbreviations: AST: aspartate aminotransferase; ALT: alanine aminotransferase; HP: hydroxyproline; HF: hepatic fibrosis; GRA: granuloma; NEC: necrosis. \# Percentage of positive staining in total liver section. 

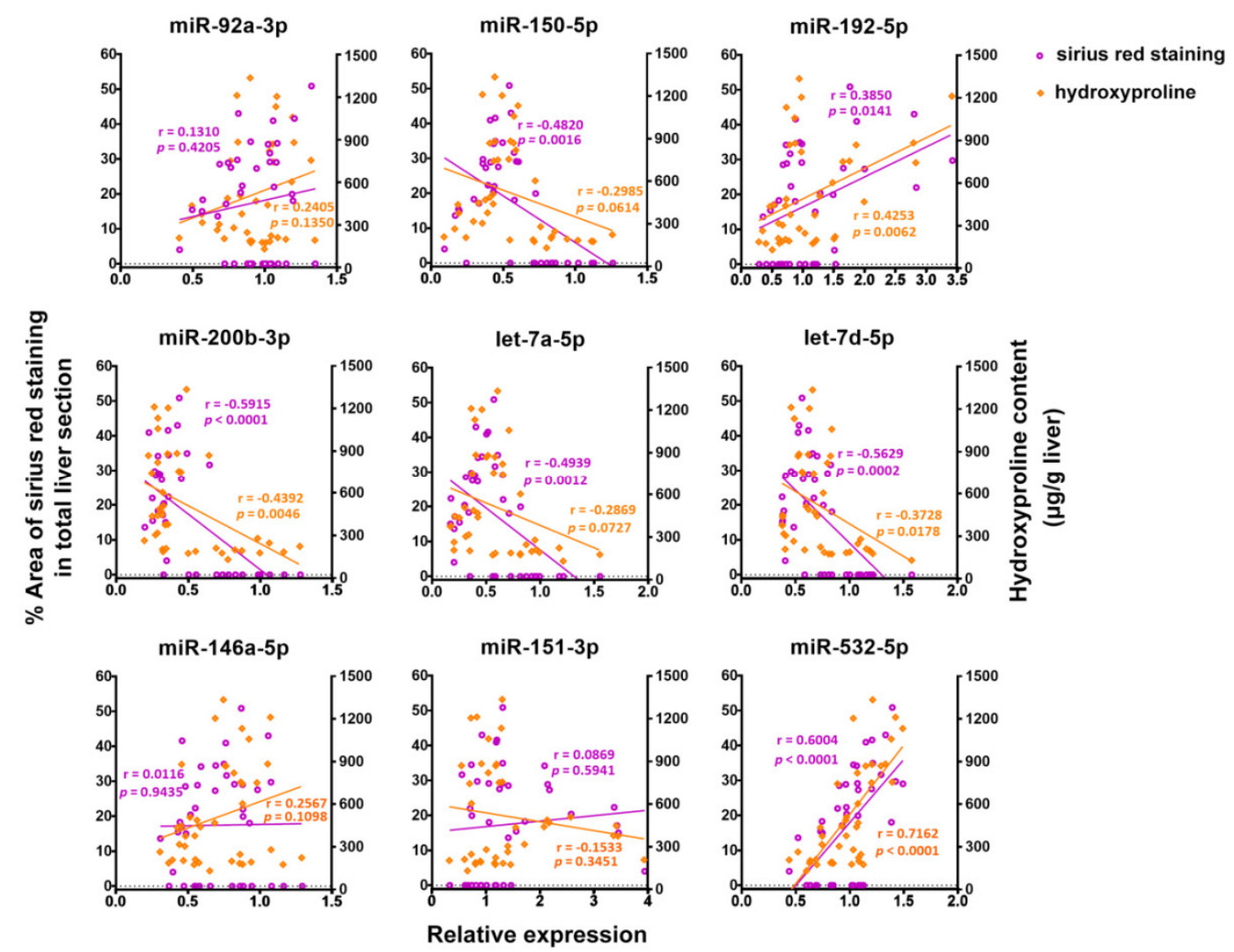

Figure 3. Correlations of serum levels of nine exomiRs with the intensities of liver fibrosis determined by sirius red staining and with liver hydroxyproline content in C57BL/6 mice during S. japonicum infection (Pearson's $r$ ).

\subsection{Identification of Endogenous RGs for Serum exomiR Expression Analysis in Schistosomiasis Patients with} Different Liver Fibrosis Grading

In order to identify suitable RGs for quantification of serum exomiRs in human schistosomiasis patients with different liver fibrosis grades, serum exosomal samples were extracted from 42 subjects resident in a schistosomiasis-endemic area. These subjects included 11, 11, 10, and 10 individuals with liver fibrosis grade 0, I, II, and III, respectively [25]. Then, the expression levels of the selected 12 candidate RGs in the serum exosomal samples were quantified by RT-PCR. Gene expression stability was then analyzed using the algorithms referred to above to rank the RGs (Figure 4). NormFinder identified miR-425-5p (0.222), let-7a-5p (0.402), and miR-16-5p (0.405) as the top three most stable candidates (Figure 4a). The geNorm ranked miR-26a-5p (0.272), miR-191-5p (0.272), and miR-103a-3p (0.312) as being among the most stable RGs (Figure $4 b$ ). Using BestKeeper analysis, miR-631 (0.404), miR-101-3p (0.535), and miR-423-5p (0.558) showed the lowest variation (Figure 4c). Using the Delta Ct method, miR-423-5p (0.6) was the most reliable, followed by miR-191-5p (0.656) and let-7a-5p (0.66) (Figure $4 \mathrm{~d}$ ). The geometric mean of all four of the methods was used to yield the final list of RG candidates, which identified miR-425-5p (2.236), miR-191-5p (2.913), and let-7a-5p (3.722) as being among the most stable genes, with miR-101-3p, miR-221-3p, and miR-181a-5p being the least stable species (Figure 4e). 

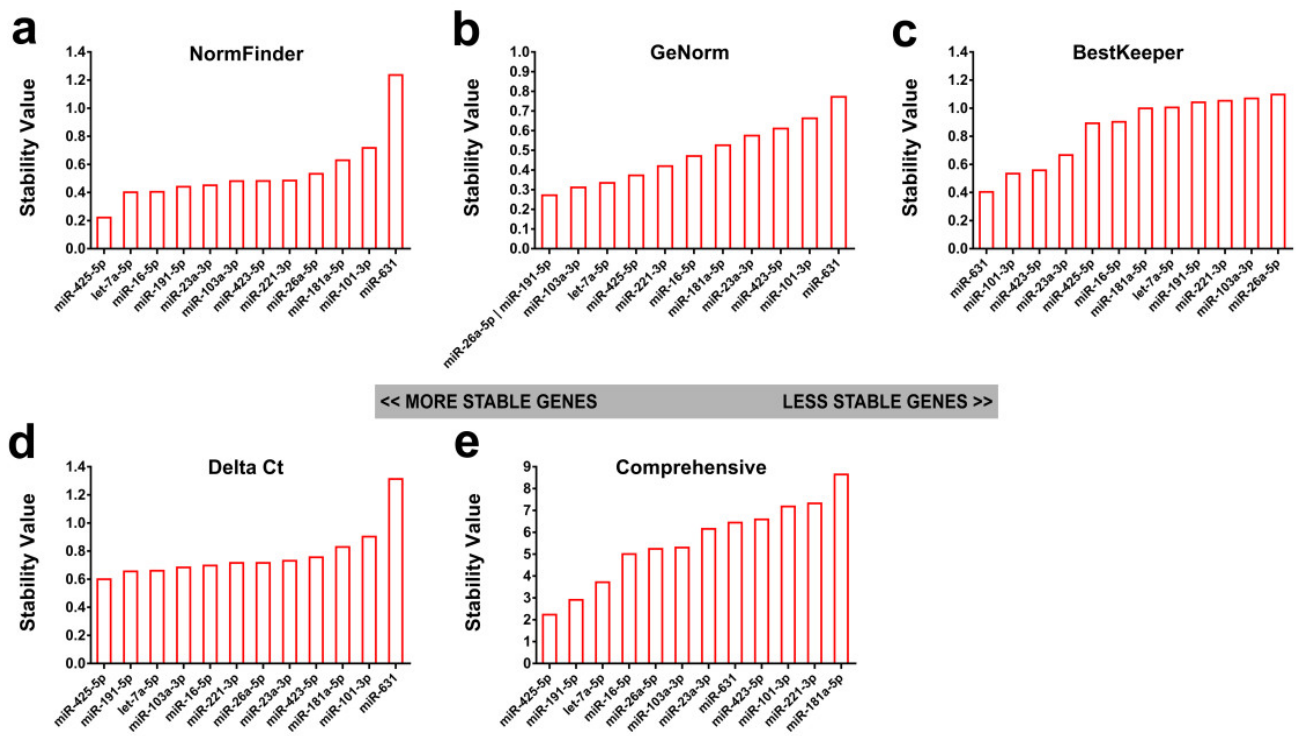

Figure 4. Identification of candidate RGs from qRT-PCR quantification data of 12 serum exomiRs in 42 subjects from a schistosomiasis-endemic area. Data were analyzed using (a) NormFinder, (b) GeNorm, (c) BestKeeper, and (d) Delta Ct. (e) A comprehensive ranking of candidate RGs was produced by RefFinder by integrating the results of the above four algorithms.

2.5. Correlations of the Serum Levels of Eleven exomiRs with the Grades of Hepatic Fibrosis in a Cohort of Subjects from a Schistosomiasis Endemic Area

Using the most stable exomiR (miR-425-5p) as reference, we analyzed the correlations of the serum levels of the other eleven RGs with hepatic fibrosis grades. A positive correlation was confirmed between the serum levels of the two exomiRs (miR-103a-3p and miR-181a-5p) with the grades of liver fibrosis within the 42 clinical samples tested for the identification of RGs (Figure 5).
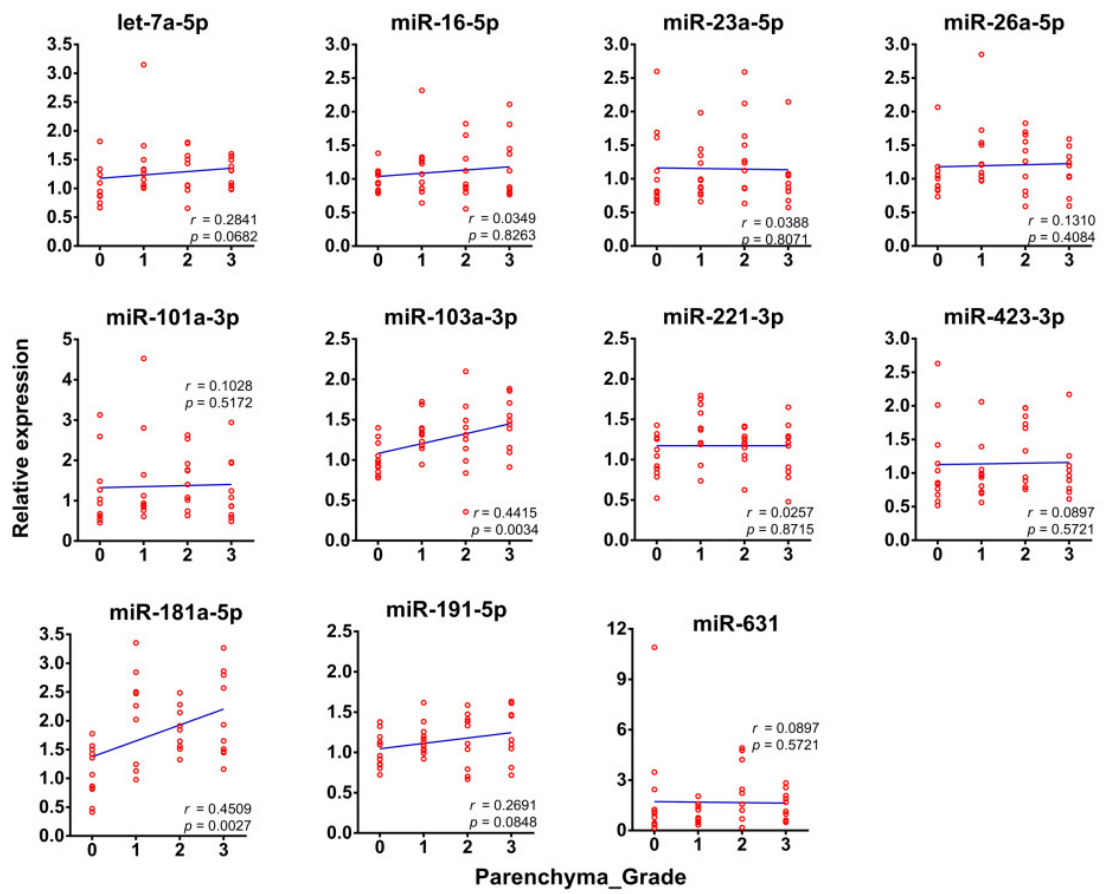

Figure 5. Correlations of the levels of the eleven exomiRs in serum (using the most stable exomiR miR-425 as reference) with hepatic fibrosis grades in the cohort subset employed for endogenous RGs identification (Spearman's correlation coefficient). 
These two exomiRs along with the initially selected nine exomiRs and were further validated with a cohort of schistosomiasis japonica patients $(n=104)$ recruited from an endemic area of the Philippines (Table 4). The potential associations between the levels of the nine exomiR signatures in serum and the grades of liver fibrosis were then assessed. The serum levels of two exomiRs miR-146a-5p and miR-532-5p were inversely correlated with liver fibrosis grades $(r=-0.2524$ and -0.2365 , and $p=0.0097$ and 0.0156 , respectively), whereas the others did not significantly correlate with hepatic fibrosis grading (Figure 6).

Table 4. The infection status and intensity of the cohort stratified by fibrosis grade.

\begin{tabular}{lcccc}
\hline \multicolumn{1}{c}{ Fibrosis Grade } & $\mathbf{0}(\boldsymbol{n}=\mathbf{3 2})$ & $\mathbf{I}(\boldsymbol{n}=\mathbf{2 7})$ & II $(\boldsymbol{n}=\mathbf{2 6})$ & III $(\boldsymbol{n}=\mathbf{1 9})$ \\
\hline M/F & $12 / 20$ & $22 / 5$ & $21 / 6$ & $19 / 0$ \\
Age (years) & $36.69 \pm 18.11$ & $35.59 \pm 15.94$ & $46.19 \pm 13.43$ & $48.32 \pm 13.25$ \\
\hline Kato-Katz test (EPG range) & $(0-220)$ & $(0-423)$ & $(0-633)$ & $(0-747)$ \\
\hline Negative (0) & 27 & 17 & 15 & 6 \\
Mild (1-99) & 4 & 6 & 8 & 10 \\
Moderate (100-399) & 1 & 3 & 2 & 2 \\
Heavy (>400) & 0 & 1 & 1 & 1 \\
\hline Serological test (+/-) & $14 / 18$ & $24 / 3$ & $25 / 1$ & $19 / 0$ \\
\hline
\end{tabular}

* Based on an ELISA assay detecting IgG antibodies against a combination of recombinant $S$. japonicum antigens (SjSAP4 plus Sj23-LHD) [26]. EPG: Egg per gram feces.
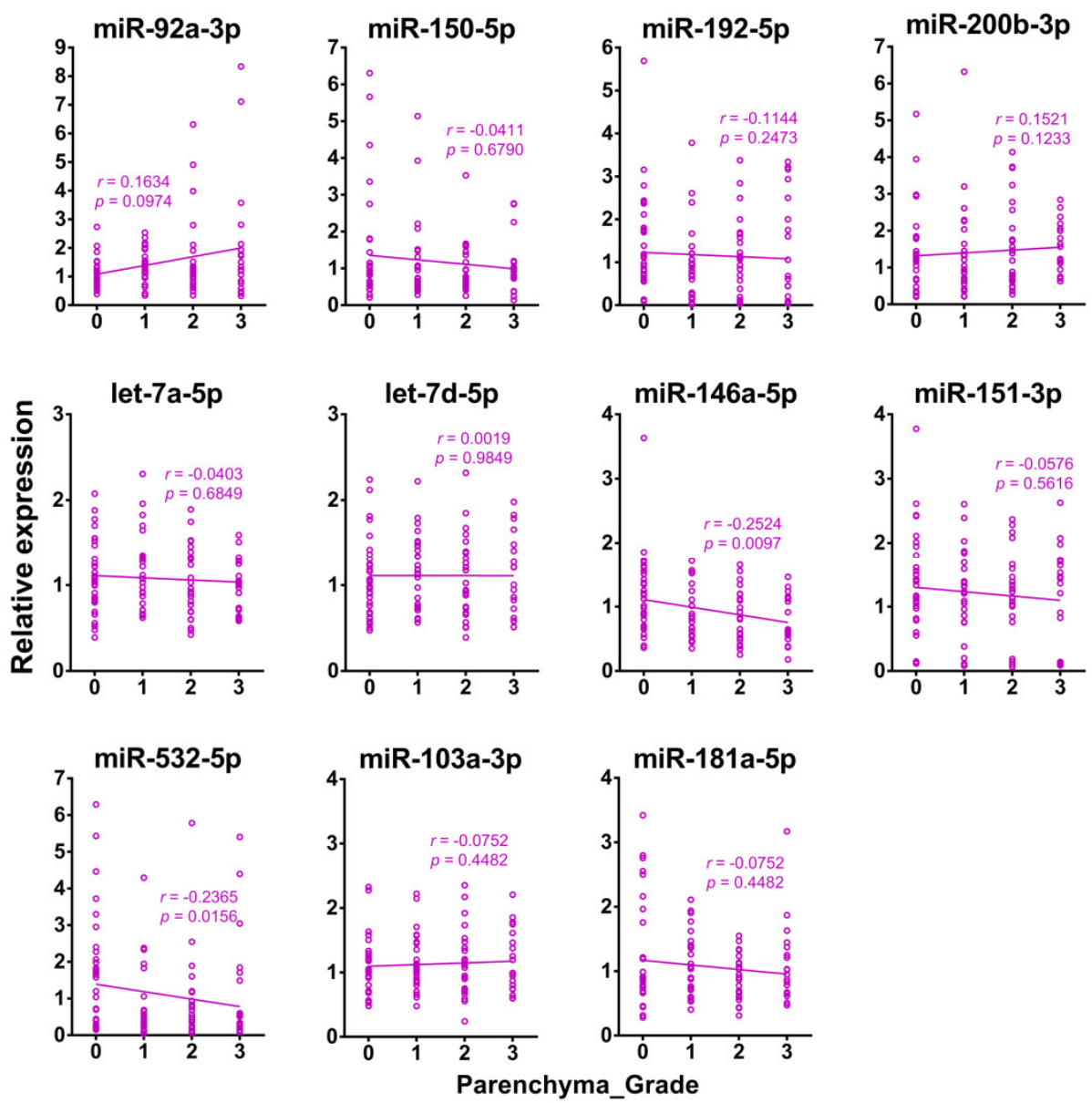

Figure 6. Correlations of the levels of eleven exomiRs in serum with hepatic fibrosis grades within the study cohort (Spearman's $r$ ). 


\subsection{Serum-Derived exomiRs Can Discriminate Different Liver Fibrosis Grades}

The levels of three exomiRs, miR-92a-3p, miR-146a-5p, and miR-532-5p, in serum exhibited a significant difference between subjects with fibrosis grades I-III compared with those without fibrosis $(p=0.0311,0.0325$, and 0.0020 , respectively) (Figure 7a), while the levels of the other eight exomiRs did not show any significant difference between the two groups. The receiver operating characteristic (ROC) analysis for discriminating liver fibrosis grades I-III from grade 0 showed that the area under the curve (AUC) levels of the three exomiRs, miR-92a-3p, miR-146a-5p, and miR-532-5p, were 0.6326, 0.6315 , and 0.6884 , respectively ( $p=0.0315,0.0329$, and 0.0023 , respectively) (Figure $7 \mathrm{~b}$ ). The best diagnostic performance was obtained with a combination of exosomal miR-146a-5p and miR-532-5p, which showed an AUC value of $0.6962(p=0.0015)$ (Figure $7 b)$. For discriminating liver fibrosis grades II-III from 0-I, we observed the serum level of exosomal miR-146a-5p or a combination of exosomal miR-146a-5p and miR-532-5p was significantly higher in individuals with mild fibrosis (grades 0-I) compared with those with severe disease (grades II-III) ( $p=0.0227$ and 0.0364 , respectively) (Figure 8a), with an AUC of 0.6305 and 0.6200 , respectively ( $p=0.0231$ and 0.0367 , respectively) (Figure 8b). No exomiRs tested were able to discriminate subjects with liver fibrosis grades 0-II from those with grade III (data not shown).
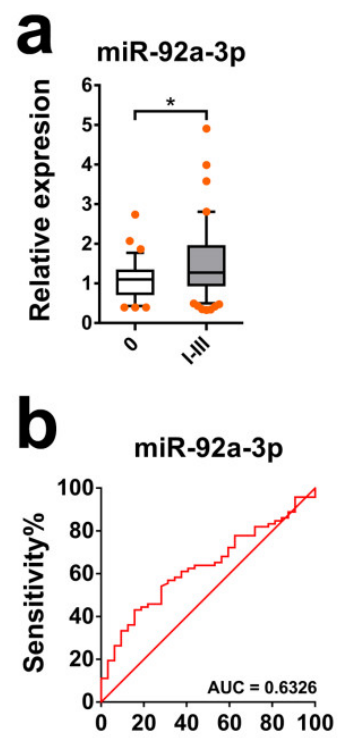

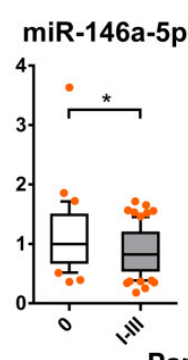

Parench

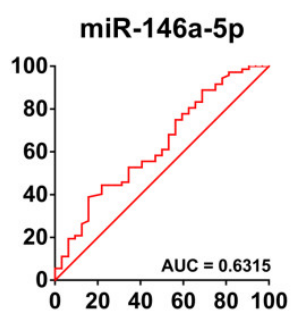

$100 \%$ - Specificity $\%$
miR-532-5p miR-146a-5p + miR-532-5p
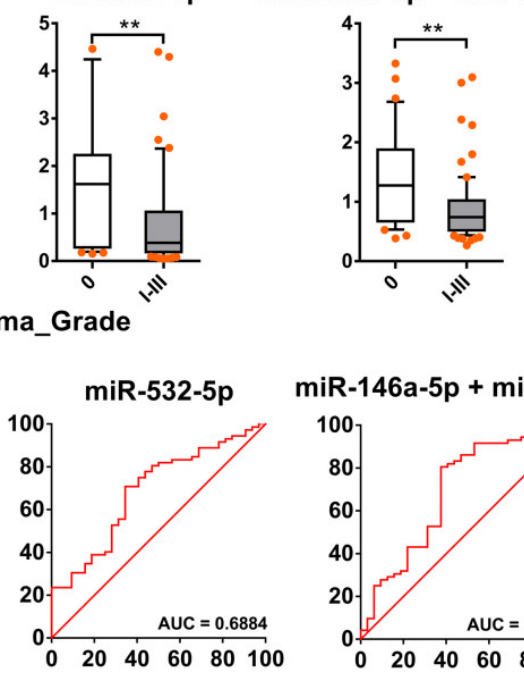

$m i R-146 a-5 p+m i R-532-5 p$

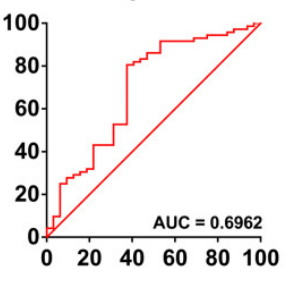

Figure 7. Discrimination of schistosomiasis hepatic fibrosis grades I-III vs. 0 by levels of exomiRs in serum. (a) The serum levels of exomiR candidates individually or in combination in subjects with (grades I-III) or without (grade 0) liver fibrosis. Boxes represent the interquartile range of the data with lines across the boxes indicating the median values. The hash marks above and below the boxes indicate the 90th and 10th percentiles, respectively. $p$ values were calculated using a Mann-Whitney U test ${ }^{*}=p<0.05,{ }^{* *}=p<0.01$ ). (b) The area under the curve (AUC) values were calculated using receiver operating characteristic (ROC) analysis to access capabilities of individual exomiR candidates or in combination in serum for discriminating subjects with stage I-III to those without liver fibrosis (grade 0$)$. 


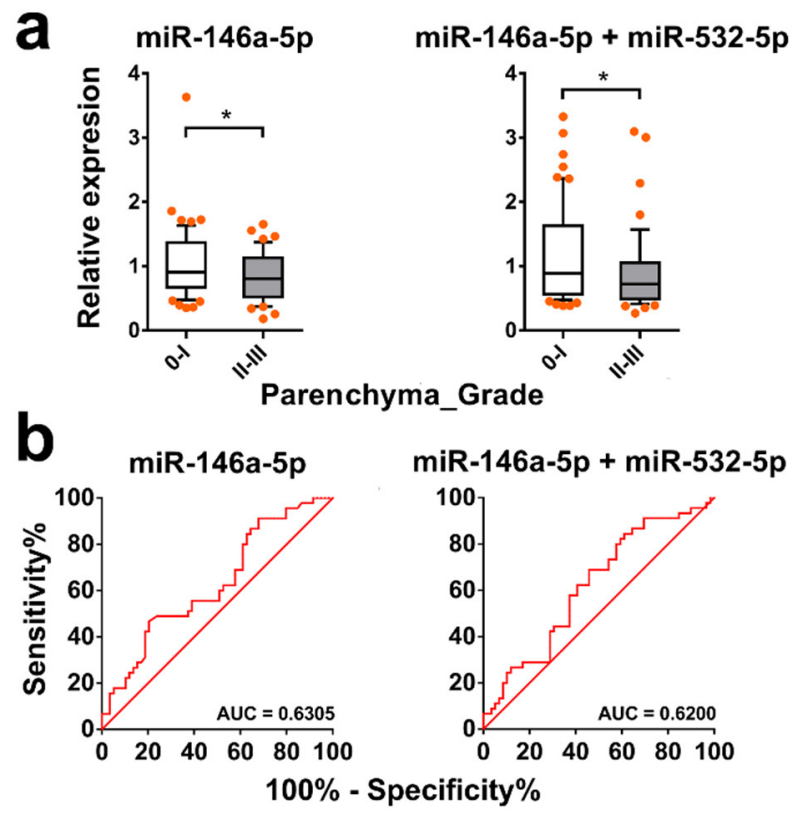

Figure 8. Discrimination of schistosomiasis hepatic fibrosis grades II-III vs. 0-I by serum levels of exomiRs. (a) The level of serum exosomal miR-146b-5p or a combination of exosomal miR-146a-5p and miR-532-5p in subjects with grades 0-I or II-III liver fibrosis. Boxes represent the interquartile range of the data with lines across the boxes indicating the median values. The hash marks above and below the boxes indicate the 90th and 10th percentiles, respectively. $p$ values were calculated using a Mann-Whitney U test $\left({ }^{*}=p<0.05\right)$. (b) The AUC values were calculated using ROC analysis to access capabilities of exomiR candidates individually or in combination in serum for discriminating subjects with severe fibrosis grades (II-III) from those without significant fibrosis (grades 0-I).

\section{Discussion}

Liver fibrosis in schistosomiasis occurs during the development of a series of complex hepatopathologies, involving immune inflammation, granuloma formation, and liver damage [4], a scenario that may be more complicated than the etiology of other chronic liver diseases, such as those due to viral infections, alcoholic hepatitis, or non-alcoholic steatohepatitis. The current study aimed to determine whether the levels of exosome-derived miRNAs in serum are able to grade the intensities of liver fibrosis due to schistosomiasis. Identification of suitable RGs is a prerequisite for the quantification of miRNAs using qRT-PCR $[27,28]$. In this study, different exomiRs were identified as the most stable genes in a murine schistosomiasis model and within clinical samples. Across the infection course in C57BL/6 mice, exosomal miR-103a-3p was identified as the as the most stable RG, while exosomal miR-425-5p was determined as the most stable RG in patients infected with S. japonicum. Previous work recommended miR-103a-3p as a suitable RG for analysis of plasma miRNAs in a murine model with acetaminophen-induced hepatotoxicity [29]. Furthermore, miR-103a-3p was determined as the second stably expressed reference gene by the geNorm and NormFinder algorithms for analyzing serum exosomal miRNA expression in patients with hepatitis B or hepatocellular carcinoma [30]. On the other hand, miR-425-5p was identified as the most stable expressed miRNA in plasma of patients with vulvar intraepithelial neoplasia lesions and vulvar carcinoma [31] and in human plasma exosomes [21]. In the murine schistosomiasis model, the experimental time frame employed (4-11 weeks p.i.) represents an acute toxemic phase, which is characterized by complicated hepatic pathologies, including necrosis, inflammation and granuloma formation within a short period [32]. We noted in this study that the levels of several miRNA signatures in the circulatory system were not only associated with the severity of liver fibrosis but also with the extent of liver damage and the degree of granulomatous involvement (Table 3), features also evident in a previous study we undertook by directly measuring serum miRNAs in two murine models of schistosomiasis [9]. In contrast, schistosome infection in the human host tends 
to be chronic [9]. Thus, the animal model does not precisely represent the pathological status of human schistosomiasis, a feature that further highlights the importance of identification of suitable normalizers for the analysis of miRNA expression in extracellular vesicles in different disease models/conditions.

Comparative miRNAome analysis revealed different miRNA expression profiles in bovine sera and serum-derived exosomes [33]. Although it has been suggested that RNA packaging into extracellular vesicles/exosomes is a selective process, the precise loading mechanism for this remains elusive [34,35]. It is not surprising to observe the inconsistent performance of specific miRNA signatures derived from serum and serum exosomes as biomarkers for a particular disease. For example, Lambrecht et al. reported that levels of vesicle-associated miRNA-192, -200b-3p, -92a-3p, and -150-5p in plasma showed diagnostic power in discriminating early stage liver fibrosis, but this was not the case when these markers were directly measured in plasma [8]. In contrast, Matsuura et al. [7] showed that the plasma levels of let-7 family members significantly declined over the progression of fibrosis in patients with chronic hepatitis C, with an AUC value between 0.734 and 0.790; however, the detection power was reduced when targeting the exosome-derived let-7 family members. Similarly, we showed previously that the serum levels of miR-150-5p, let-7a-5p, and let-7d-5p in patients were able to discriminate mild from severe hepatic fibrosis caused by schistosome infection, with an AUC value of $0.6838,0.6598$, and 0.6270 , respectively [9]. And, here, we found that the levels of these three miRNAs in serum exosomes were not capable of discriminating liver fibrosis grades 0 versus I-III, nor grades $0-1$ versus II-III or grades 0 -II versus III in the human subjects tested. Nevertheless, when compared with our previous study [9], similar trends were evident in the expression levels of several miRNAs (i.e., miR-150-5p, miR-192-5p, miR-200b-3p, let-7a-5p, let-7d-5p, and miR-146a-5p) in serum or in serum exosomes in C57BL/6 mice during $S$. japonicum infection.

It has been recently documented that miR-146a-5p participates in the biogenesis of fibrosis in a variety of tissues [36-40]. In the pathogenesis of nonalcoholic fibrosing steatohepatitis, it has been shown that miR-146a-5p was significantly down-regulated in activated hepatic stellate cells (HSCs) and overexpression of miR-146a-5p suppressed HSC activation, as well as extracellular matrix deposition [41]. During the development of liver fibrosis in a murine model of schistosomiasis, He et al. found that by targeting Signal Transducer And Activator Of Transcription 1 (STAT1), miR-146 suppressed the interferon (IFN)- $\gamma$-induced differentiation of macrophages to M1 cells [38]. In relation to the application of extracellular miR-146a-5p in staging liver fibrosis, Appourchaux et al. found that the serum level of miR-146a-5p was higher in chronic hepatitis $C$ patients with advanced fibrosis and cirrhosis (F3-F4) than those with mild and moderate fibrosis (F1-F2); in contrast, no difference in the serum level of miR-146a-5p was observed between chronic hepatitis B patients with F3-F4 cirrhosis and those with F1-F2 [42]. In the current study, consistent with our previous study of direct measurement of serum miR-146a-5p [9], down-regulation of exosomal miR-146a-5p in the sera of C57BL/6 mice was observed over the course of $S$. japonicum infection, although no direct correlation between the serum level of exosomal miR-146a-5p and HP or HF was found. Furthermore, a negative correlation between the serum level of this miRNA and the grades of liver fibrosis was observed in the human cohort we investigated, which enables the discrimination of individuals with moderate to severe (grade II-III) liver fibrosis from those with mild (grade $0-\mathrm{I}$ ) disease. Nevertheless, there is still a concern that using a single miRNA/exomiR marker in the circulatory system for predicting a particular disease may incur a potential problem of non-specificity. For example, it has been shown recently that the level of serum exosomal miR-146a-5p declined significantly in systemic lupus erythematosus patients compared with healthy controls [43]. Accordingly, the use of a combination of multiple miRNA/exomiR signatures may improve diagnostic specificity.

Recent reports indicate that miR-532 family members are dysregulated in various tumors and are associated with carcinogenesis, thus having been considered as potential novel therapeutic interventions for the treatment of specific tumors [44-46]. Furthermore, Chen et al. found that serum EV miR-532-5p was significantly higher in control subjects versus F3/F4 fibrosis patients, using U6 as a reference gene [24]. Here, we showed that exosomal miR-532-5p inversely correlated with liver fibrosis grades 
in the human schistosomiasis cohort and was able to discriminate hepatic fibrosis I-III from those without fibrosis (AUC: 0.6884). However, in C57BL/6 during S. japonicum infection, a strong positive correlation was observed between the levels of serum exosomal miR-532-5p with the grades of liver fibrosis, which further echoed the different pathological severities in the murine schistosomiasis model, which usually represents a high dosage infection, compared with schistosome-infected patients.

Moderate diagnostic values were obtained in the grading of schistosomal liver fibrosis by detection of an individual exomiR or in combination. For discriminating individuals without liver fibrosis from those with grades I-III, the best diagnostic performance was obtained with the combination of serum exosomal miR-146a-5p and miR-532-5p (AUC: 0.6962, $p=0.0015$ ) (Figure 7b). For discriminating mild from severe liver fibrosis, the diagnostic performance of exosomal miR-146a-5p in serum was not superior but comparable with that of miRNAs directly isolated from serum, i.e., AUCs of 0.6575 and 0.6305 were obtained for serum miR-146a-5p and serum exosomal miR-146a-5p, respectively. The following factors may contribute to this phenomenon: (i) It has been shown that re-infection with S. japonicum and co-parasitism with intestinal helminths and protozoa were common in the cohort subjects investigated $[47,48]$, a feature which potentially might alter the host immune responses and further dysregulate the serum levels of the targeted exomiRs; (ii) as the study cohort individuals were from a rural area with rudimentary water sources and poor sanitation and hygiene [47], acute respiratory infections, diarrheal diseases, and other communicable diseases are likely to have had a considerable influence on the expression of the exomiRs investigated; (iii) only a limited number of exomiRs were tested in the current study, and it would be informative to identify more potential exomiRs specific for schistosomiasis fibrosis by profiling; (iv) importantly, not only the liver, but also other organs, i.e., the intestine, spleen and other ectopic sites (such as kidney and brain), are likely affected during hepatosplenic schistosomiasis [49]. Since serum exosomes can be released from all affected organs, the pathological alternation in multiple organs further likely changes the levels of exomiRs tested. Thus, assessment of miRNAs from sub-population exosomes, such as liver-derived [50] or, more specifically, HSCs-derived exosomes [51], may represent a promising pipeline to improve diagnostic power, as has been suggested for improving the performance of tumor diagnosis by tagging tumor-derived exomiRs in the blood circulation [52]. In this regard, membrane markers for liver-specific or HSC-specific exosomes need to be identified prior to applying this approach in the discovery of biomarkers for liver fibrosis or other liver diseases [53,54].

\section{Materials and Methods}

\subsection{Ethics Statement}

This study was approved by the QIMR Berghofer Medical Research Institute (QIMRB) Human Ethics Committee and the Ethics Committee of the Research Institute for Tropical Medicine (RITM), Manila, approved the study protocol in accordance with the Declaration of Helsinki (Institutional Review Board Number 2012-13-0, approved on 07/05/2012). Written informed consent was received from each study participant or from the legal guardians of those aged less than 15 years.

\subsection{Animal Infection, Serum Collection, Histological Assessment, and Biochemical Analyses}

Eight-week-old female C57BL/6 mice were divided into 6 groups and were percutaneously infected with $16 \pm 2$ of $S$. japonicum cercariae. Subsequently, mice were sacrificed at 0 (uninfected), $4,6,7,9$, and 11 weeks post-infection, and $\sim 1 \mathrm{~mL}$ blood was collected from each mouse at each time point by cardiac puncture. The median lobe was collected from each mouse liver and subjected to histological assessment after formalin fixation [32]. Slides were scanned using the Aperio Slide Scanner (Aperio Technologies, Vista, CA, USA). Liver hydroxyproline (HP) content was assessed by a hydroxyproline colorimetric assay kit (Bioo Scientific, Austin, TX, USA). Serum alanine transaminase (ALT) and aspartate transaminase (AST) levels were tested using ALT and AST color endpoint assay kits (Bioo Scientific), respectively. 


\subsection{Study Cohort}

The human cohort was recruited from 18 schistosomiasis-endemic but non-malaria-endemic barangays in the municipalities of Laoang and Palapag, Northern Samar, the Philippines in 2012. For more than over the last three decades, the area has had active schistosomiasis control programs, including a mass drug administration (MDA) program which commenced in 2008. Free annual treatment (Praziquantel $40 \mathrm{mg} / \mathrm{kg}$ in a single dose) is delivered to all individuals aged between 5-65 years in accordance with the Department of Health Administrative Order 2007-0015, but the compliance rate varies [55]. It is worthwhile to note that the S. japonicum reinfection rate in the area is also high [56]. Detailed information of this study cohort is described elsewhere as part of a hepatic morbidity study [25,47]. In brief, Kato-Katz thick smear stool examination was performed on each individual in the cohort using two stool specimens provided over the course of a week to determine infection status and intensity. Six Kato-Katz thick smears were prepared on microscope slides using standard $50 \mathrm{mg}$ templates according to established protocols and examined under a light microscope by experienced technicians. All subjects in the cohort were assessed for liver fibrosis severity by ultrasound scan using a portable gray-scale ultrasonogram (SONOACE X1; Madison Co., Ltd., Seoul, South Korea) [25]. The parenchymal pattern of hepatic fibrosis was determined according to practical guidelines recommended by the WHO for ultrasonographic examination for schistosomiasis-related morbidity [57]. In the current study, 104 individuals (all negative for hepatitis B virus/hepatitis $C$ virus infection) from the cohort were enrolled. For serum collection, $\sim 10 \mathrm{~mL}$ blood was drawn from each subject enrolled; serum was subsequently separated by centrifugation, and stored at $2-8{ }^{\circ} \mathrm{C}$. The serum samples were then transported to the Research Institute for Tropical Medicine (RITM), Manila, at a temperature of $4{ }^{\circ} \mathrm{C}$ and stored at $-80^{\circ} \mathrm{C}$. Subsequently, sample aliquots were transported on dry ice to QIMRB, Australia.

\subsection{Exosome Isolation, RNA Extraction, Polyadenylation, and Reverse Transcription (RT)}

Serum exosomes were isolated using ExoQuick reagent (SBI System Biosciences, Palo Alto, CA, USA), according to the manufacturer's protocol. Briefly, one hundred microliters of mouse or human serum was mixed with $31 \mu \mathrm{L}$ of ExoQuick exosome precipitation solution. After incubation at $4{ }^{\circ} \mathrm{C}$ for $30 \mathrm{~min}$, the samples were then centrifuged at $1500 \times g$ for $30 \mathrm{~min}$. The supernatant was removed, and the exosome-rich pellet was re-suspended by adding $100 \mu \mathrm{L}$ PBS. Subsequently, total RNA was extracted from the re-suspended samples using a Qiagen miRNeasy Mini Kit (Qiagen, Hilden, Germany), following the manufacturer's instruction. RNA concentration was quantified using a Qubit MicroRNA Assay Kit (Thermo Fisher Scientific, Waltham, MA, USA). Polyadenylation and RT reactions were performed using the S-Poly $(\mathrm{T})$ method in a one-step procedure by the combined use of two commercial kits: the Poly(A) Polymerase Tailing Kit (Lucigen, Middleton, WI, USA) and the TaqMan MicroRNA Reverse Transcription Kit (Thermo Fisher Scientific, Waltham, MA, USA) [32,58]. One ng RNA (5 $\mu$ L) was used in each RT reaction, which was carried out under the following condition: $37^{\circ} \mathrm{C}$ for $30 \mathrm{~min}$, $42{ }^{\circ} \mathrm{C}$ for $30 \mathrm{~min}$, and $85^{\circ} \mathrm{C}$ for $5 \mathrm{~min}$. RT products were stored at $-20^{\circ} \mathrm{C}$ prior to subsequent analysis. The RT primers used in this study are listed in Supplementary Tables S1-S3.

\subsection{QRT-PCR for miRNA Quantification}

Quantification of miRNAs was performed by qRT-PCR with the Applied Biosystems Quantstudio 5 Real-Time PCR System (Thermo Fisher Scientific, Waltham, MA, USA) according essentially to protocols described previously [32]. The assays were performed with the following cycling conditions: $50{ }^{\circ} \mathrm{C}, 2 \mathrm{~min}, 95^{\circ} \mathrm{C} 10 \mathrm{~min}$, followed by 40 cycles: $95^{\circ} \mathrm{C}$ for $15 \mathrm{~s}$, and $60^{\circ} \mathrm{C}$ for $1 \mathrm{~min}$. The expression levels of miRNAs were calculated by the $2^{-\Delta \Delta C t}$ method with normalization to the reference genes identified in this study for analysis [32]. 


\subsection{Reference Genes Identification}

To identify RGs used for the analysis of exomiR expression in serum for predicting the severity of liver fibrosis, we evaluated two different data sets: (i) the temporal expression data of candidate RGs in a murine model during S. japonicum infection; (ii) the expression data of candidate RGs in serum samples of 42 subjects from a schistosomiasis-endemic area with different liver fibrosis grades. The relative expression stability of the candidates was analyzed using four computational programs (NormFinder [59], GeNorm [60], BestKeeper [61], and the comparative Delta Ct method [62]), which are collectively available at a user-friendly web-based RefFinder tool [63] (https://www.heartcure. com.au/reffinder). A recommended comprehensive ranking of candidate RGs was then generated by RefFinder based on the results of the four algorithms.

\subsection{Statistical Analysis}

For analysis of the temporal levels of serum exomiRs in C57BL/6 mice during S. japonicum infection, one-way ANOVA was used followed by the Holm-Sidak multiple comparison. Pearson's correlation coefficient $(r)$ was used for the determination of the correlations between continuous variables, while Spearman's rank correlation coefficient (rho) was employed for the assessment of the correlations between continuous and categorical variables. The Mann-Whitney $U$-test was used for analysis of the ability of the levels of serum exomiRs in discriminating different schistosomiasis liver fibrosis grades. Receiver operating characteristic (ROC) curve analyses were performed, and the area under the curve (AUC) was calculated to evaluate the potency of using the serum exomiRs as biomarkers for discriminating hepatic fibrosis caused by schistosomiasis. A $p$-value $<0.05$ was considered statistically significant. Statistical analysis was performed with GraphPad Prism v. 6.00 for Windows.

\section{Conclusions}

In summary, optimal reference genes were determined for studying exomiRs in a schistosomiasis murine model and a clinical cohort from a schistosomiasis endemic area, which may be of value for future research focused on serum/plasma exomiRs in fibrosis. We also identified serum exomiRs that are capable of grading hepatic fibrosis due to schistosomiasis japonica. The diagnostic performance of these exomiRs is moderate and, consequently, additional optimization steps, such as evaluating miRNAs in liver-derived or HSCs-derived exosomes [50], will be required to improve the overall diagnostic power using this approach.

Supplementary Materials: Supplementary materials can be found at http:/www.mdpi.com/1422-0067/21/10/ 3560/s1. Table S1: Primers and probe used for optimal reference gene identification; Table S2: Primers and probe used for quantification of serum exosomal miRNA candidates for grading the intensities of liver fibrosis in a murine schistosomiasis model; Table S3: Primers and probe used for quantification of serum exosomal miRNA candidates for grading liver fibrosis in a human cohort from a schistosomiasis endemic area.

Author Contributions: Conceptualization, P.C. and D.P.M.; Methodology, P.C., Y.M. and R.M.O.; Clinical sample contribution, R.M.O., A.G.R. and D.U.O.; Investigation, P.C., Y.M., R.M.O., A.G.R. and D.U.O.; Writing-Original Draft, P.C. and Y.M.; Writing-Review \& Editing, A.G.R. and D.P.M.; Supervision, P.C. and D.P.M.; Funding Acquisition, P.C. and D.P.M. All authors have read and agreed to the published version of the manuscript.

Funding: This research was funded by the National Health and Medical Research Council (NHMRC) of Australia (ID: APP1160046, APP1102926, APP1037304, and APP1098244). DPM is an NHMRC Senior Principal Research Fellow and Senior Scientist at QIMRB.

Acknowledgments: We thank Mary Duke for maintenance of the S. japonicum lifecycle at QIMR Berghofer Medical Research Institute (QIMRB). We also thank the local field and clinical staff in Palapag, Northern Samar, the Philippines, for their kind assistance in the collection of clinical samples.

Conflicts of Interest: The authors declare no conflict of interest. The funders had no role in study design, data collection and analysis, decision to publish, nor preparation of the manuscript. 


\section{References}

1. Colley, D.G.; Bustinduy, A.L.; Secor, W.E.; King, C.H. Human schistosomiasis. Lancet 2014, 383, $2253-2264$. [CrossRef]

2. McManus, D.P.; Dunne, D.W.; Sacko, M.; Utzinger, J.; Vennervald, B.J.; Zhou, X.N. Schistosomiasis. Nat. Rev. Dis. Primers 2018, 4, 13. [CrossRef] [PubMed]

3. Anthony, B.J.; Ramm, G.A.; McManus, D.P. Role of resident liver cells in the pathogenesis of schistosomiasis. Trends Parasitol. 2012, 28, 572-579. [CrossRef] [PubMed]

4. Chuah, C.; Jones, M.K.; Burke, M.L.; McManus, D.P.; Gobert, G.N. Cellular and chemokine-mediated regulation in schistosome-induced hepatic pathology. Trends Parasitol. 2014, 30, 141-150. [CrossRef]

5. Roderburg, C.; Urban, G.W.; Bettermann, K.; Vucur, M.; Zimmermann, H.; Schmidt, S.; Janssen, J.; Koppe, C.; Knolle, P.; Castoldi, M.; et al. Micro-RNA profiling reveals a role for miR-29 in human and murine liver fibrosis. Hepatology 2011, 53, 209-218. [CrossRef]

6. Cai, P.; Piao, X.; Liu, S.; Hou, N.; Wang, H.; Chen, Q. MicroRNA-gene expression network in murine liver during Schistosoma japonicum infection. PLoS ONE 2013, 8, e67037. [CrossRef]

7. Matsuura, K.; De Giorgi, V.; Schechterly, C.; Wang, R.Y.; Farci, P.; Tanaka, Y.; Alter, H.J. Circulating let-7 levels in plasma and extracellular vesicles correlate with hepatic fibrosis progression in chronic hepatitis C. Hepatology 2016, 64, 732-745. [CrossRef]

8. Lambrecht, J.; Jan Poortmans, P.; Verhulst, S.; Reynaert, H.; Mannaerts, I.; van Grunsven, L.A. Circulating ECV-associated miRNAs as potential clinical biomarkers in early stage HBV and HCV induced liver fibrosis. Front. Pharmacol. 2017, 8, 56. [CrossRef]

9. Cai, P.; Mu, Y.; Olveda, R.M.; Ross, A.G.; Olveda, D.U.; McManus, D.P. Circulating miRNAs as footprints for liver fibrosis grading in schistosomiasis. EBioMedicine 2018, 37, 334-343. [CrossRef]

10. Nallagangula, K.S.; Nagaraj, S.K.; Venkataswamy, L.; Chandrappa, M. Liver fibrosis: A compilation on the biomarkers status and their significance during disease progression. Future Sci. OA 2017, 4, FSO250. [CrossRef]

11. Pang, B.; Zhu, Y.; Ni, J.; Thompson, J.; Malouf, D.; Bucci, J.; Graham, P.; Li, Y. Extracellular vesicles: The next generation of biomarkers for liquid biopsy-based prostate cancer diagnosis. Theranostics 2020, 10, 2309-2326. [CrossRef]

12. Desmond, B.J.; Dennett, E.R.; Danielson, K.M. Circulating extracellular vesicle microRNA as diagnostic biomarkers in early colorectal cancer-A review. Cancers 2020, 12, 52. [CrossRef]

13. Wang, S.; Wang, J.-Q.; Lv, X.-W. Exosomal miRNAs as biomarkers in the diagnosis of liver disease. Biomark. Med. 2017, 11, 491-501. [CrossRef] [PubMed]

14. Das, S.; Ansel, K.M.; Bitzer, M.; Breakefield, X.O.; Charest, A.; Galas, D.J.; Gerstein, M.B.; Gupta, M.; Milosavljevic, A.; McManus, M.T.; et al. The extracellular RNA communication consortium: Establishing foundational knowledge and technologies for extracellular RNA research. Cell 2019, 177, 231-242. [CrossRef] [PubMed]

15. Becker, A.; Thakur, B.K.; Weiss, J.M.; Kim, H.S.; Peinado, H.; Lyden, D. Extracellular vesicles in cancer: Cell-to-cell mediators of metastasis. Cancer Cell 2016, 30, 836-848. [CrossRef] [PubMed]

16. Donati, S.; Ciuffi, S.; Brandi, M.L. Human circulating miRNAs real-time qRT-PCR-based analysis: An overview of endogenous reference genes used for data normalization. Int. J. Mol. Sci. 2019, 20, 4353. [CrossRef] [PubMed]

17. Li, Y.; Xiang, G.M.; Liu, L.L.; Liu, C.; Liu, F.; Jiang, D.N.; Pu, X.Y. Assessment of endogenous reference gene suitability for serum exosomal microRNA expression analysis in liver carcinoma resection studies. Mol. Med. Rep. 2015, 12, 4683-4691. [CrossRef]

18. Li, Y.; Zhang, L.; Liu, F.; Xiang, G.; Jiang, D.; Pu, X. Identification of endogenous controls for analyzing serum exosomal miRNA in patients with hepatitis B or hepatocellular carcinoma. Dis. Markers 2015, 2015, 12. [CrossRef]

19. Ragni, E.; Perucca Orfei, C.; De Luca, P.; Colombini, A.; Vigano, M.; Lugano, G.; Bollati, V.; de Girolamo, L. Identification of miRNA reference genes in extracellular vesicles from adipose derived mesenchymal stem cells for studying osteoarthritis. Int. J. Mol. Sci. 2019, 20, 1108. [CrossRef] 
20. Gouin, K.; Peck, K.; Antes, T.; Johnson, J.L.; Li, C.; Vaturi, S.D.; Middleton, R.; de Couto, G.; Walravens, A.-S.; Rodriguez-Borlado, L.; et al. A comprehensive method for identification of suitable reference genes in extracellular vesicles. J. Extracell. Vesicles 2017, 6, 1347019. [CrossRef]

21. Santovito, D.; De Nardis, V.; Marcantonio, P.; Mandolini, C.; Paganelli, C.; Vitale, E.; Buttitta, F.; Bucci, M.; Mezzetti, A.; Consoli, A.; et al. Plasma exosome microRNA profiling unravels a new potential modulator of adiponectin pathway in diabetes: Effect of glycemic control. J. Clin. Endocrinol. Metab. 2014, 99, E1681-E1685. [CrossRef] [PubMed]

22. Garcia-Contreras, M.; Shah, S.H.; Tamayo, A.; Robbins, P.D.; Golberg, R.B.; Mendez, A.J.; Ricordi, C. Plasma-derived exosome characterization reveals a distinct microRNA signature in long duration Type 1 diabetes. Sci. Rep. 2017, 7, 5998. [CrossRef] [PubMed]

23. Matsuura, K.; Aizawa, N.; Enomoto, H.; Nishiguchi, S.; Toyoda, H.; Kumada, T.; Iio, E.; Ito, K.; Ogawa, S.; Isogawa, M.; et al. Circulating let-7 levels in serum correlate with the severity of hepatic fibrosis in chronic hepatitis C. Open Forum Infect. Dis. 2018, 5, ofy268. [CrossRef] [PubMed]

24. Chen, L.; Chen, R.; Kemper, S.; Cong, M.; You, H.; Brigstock, D.R. Therapeutic effects of serum extracellular vesicles in liver fibrosis. J. Extracell. Vesicles 2018, 7, 1461505. [CrossRef] [PubMed]

25. Olveda, D.U.; Inobaya, M.; Olveda, R.M.; Vinluan, M.L.; Ng, S.K.; Weerakoon, K.; McManus, D.P.; Ramm, G.A.; Harn, D.A.; Li, Y.; et al. Diagnosing schistosomiasis-induced liver morbidity: Implications for global control. Int. J. Infect. Dis. 2017, 54, 138-144. [CrossRef]

26. Cai, P.; Weerakoon, K.G.; Mu, Y.; Olveda, D.U.; Piao, X.; Liu, S.; Olveda, R.M.; Chen, Q.; Ross, A.G.; McManus, D.P. A parallel comparison of antigen candidates for development of an optimized serological diagnosis of schistosomiasis japonica in the Philippines. EBioMedicine 2017, 24, 237-246. [CrossRef]

27. Schwarzenbach, H.; da Silva, A.M.; Calin, G.; Pantel, K. Data normalization strategies for microRNA quantification. Clin. Chem. 2015, 61, 1333-1342. [CrossRef] [PubMed]

28. Wang, L.; Liu, Y.; Du, L.; Li, J.; Jiang, X.; Zheng, G.; Qu, A.; Wang, H.; Wang, L.; Zhang, X.; et al. Identification and validation of reference genes for the detection of serum microRNAs by reverse transcription-quantitative polymerase chain reaction in patients with bladder cancer. Mol. Med. Rep. 2015, 12, 615-622. [CrossRef]

29. Wang, Y.; Tang, N.; Hui, T.; Wang, S.; Zeng, X.; Li, H.; Ma, J. Identification of endogenous reference genes for RT-qPCR analysis of plasma microRNAs levels in rats with acetaminophen-induced hepatotoxicity. J. Appl. Toxicol. 2013, 33, 1330-1336. [CrossRef]

30. Li, L.M.; Hu, Z.B.; Zhou, Z.X.; Chen, X.; Liu, F.Y.; Zhang, J.F.; Shen, H.B.; Zhang, C.Y.; Zen, K. Serum microRNA profiles serve as novel biomarkers for HBV infection and diagnosis of HBV-positive hepatocarcinoma. Cancer Res. 2010, 70, 9798-9807. [CrossRef]

31. Zalewski, K.; Misiek, M.; Kowalik, A.; Bakula-Zalewska, E.; Kopczynski, J.; Zielinska, A.; Bidzinski, M.; Radziszewski, J.; Gozdz, S.; Kowalewska, M. Normalizers for microRNA quantification in plasma of patients with vulvar intraepithelial neoplasia lesions and vulvar carcinoma. Tumour Biol. 2017, 39, 1010428317717140. [CrossRef] [PubMed]

32. Cai, P.; Gobert, G.N.; You, H.; Duke, M.; McManus, D.P. Circulating miRNAs: Potential novel biomarkers for hepatopathology progression and diagnosis of schistosomiasis japonica in two murine models. PLoS Negl. Trop. Dis. 2015, 9, e0003965. [CrossRef] [PubMed]

33. Zhao, K.; Liang, G.; Sun, X.; Guan, L.L. Comparative miRNAome analysis revealed different miRNA expression profiles in bovine sera and exosomes. BMC Genom. 2016, 17, 630. [CrossRef] [PubMed]

34. Janas, T.; Janas, M.M.; Sapon, K.; Janas, T. Mechanisms of RNA loading into exosomes. FEBS Lett. 2015, 589, 1391-1398. [CrossRef] [PubMed]

35. Gon, Y.; Maruoka, S.; Inoue, T.; Kuroda, K.; Yamagishi, K.; Kozu, Y.; Shikano, S.; Soda, K.; Lotvall, J.; Hashimoto, S. Selective release of miRNAs via extracellular vesicles is associated with house-dust mite allergen-induced airway inflammation. Clin. Exp. Allergy 2017, 47, 1586-1598. [CrossRef]

36. Zou, Y.; Cai, Y.; Lu, D.; Zhou, Y.; Yao, Q.; Zhang, S. MicroRNA-146a-5p attenuates liver fibrosis by suppressing profibrogenic effects of TGFbeta1 and lipopolysaccharide. Cell. Signal. 2017, 39, 1-8. [CrossRef]

37. Jang, S.Y.; Park, S.J.; Chae, M.K.; Lee, J.H.; Lee, E.J.; Yoon, J.S. Role of microRNA-146a in regulation of fibrosis in orbital fibroblasts from patients with Graves' orbitopathy. Br. J. Ophthalmol. 2018, 102, 407-414. [CrossRef]

38. He, X.; Tang, R.; Sun, Y.; Wang, Y.G.; Zhen, K.Y.; Zhang, D.M.; Pan, W.Q. MicroR-146 blocks the activation of M1 macrophage by targeting signal transducer and activator of transcription 1 in hepatic schistosomiasis. EBioMedicine 2016, 13, 339-347. [CrossRef] 
39. Feng, B.; Chen, S.; Gordon, A.D.; Chakrabarti, S. miR-146a mediates inflammatory changes and fibrosis in the heart in diabetes. J. Mol. Cell. Cardiol. 2017, 105, 70-76. [CrossRef] [PubMed]

40. Morishita, Y.; Imai, T.; Yoshizawa, H.; Watanabe, M.; Ishibashi, K.; Muto, S.; Nagata, D. Delivery of microRNA-146a with polyethylenimine nanoparticles inhibits renal fibrosis in vivo. Int. J. Nanomed. 2015, 10, 3475-3488. [CrossRef]

41. Du, J.; Niu, X.; Wang, Y.; Kong, L.; Wang, R.; Zhang, Y.; Zhao, S.; Nan, Y. MiR-146a-5p suppresses activation and proliferation of hepatic stellate cells in nonalcoholic fibrosing steatohepatitis through directly targeting Wnt1 and Wnt5a. Sci. Rep. 2015, 5, 16163. [CrossRef] [PubMed]

42. Appourchaux, K.; Dokmak, S.; Resche-Rigon, M.; Treton, X.; Lapalus, M.; Gattolliat, C.H.; Porchet, E.; Martinot-Peignoux, M.; Boyer, N.; Vidaud, M.; et al. MicroRNA-based diagnostic tools for advanced fibrosis and cirrhosis in patients with chronic hepatitis B and C. Sci. Rep. 2016, 6, 34935. [CrossRef] [PubMed]

43. Dong, C.; Zhou, Q.; Fu, T.; Zhao, R.; Yang, J.; Kong, X.; Zhang, Z.; Sun, C.; Bao, Y.; Ge, X.; et al. Circulating exosomes derived-miR-146a from systemic lupus erythematosus patients regulates senescence of mesenchymal stem cells. Biomed. Res. Int. 2019, 2019, 6071308. [CrossRef] [PubMed]

44. Xie, X.; Pan, J.; Han, X.; Chen, W. Downregulation of microRNA-532-5p promotes the proliferation and invasion of bladder cancer cells through promotion of HMGB3/Wnt/beta-catenin signaling. Chem. Biol. Interact. 2019, 300, 73-81. [CrossRef]

45. Huang, L.; Tang, X.; Shi, X.; Su, L. miR-532-5p promotes breast cancer proliferation and migration by targeting RERG. Exp. Ther. Med. 2020, 19, 400-408. [CrossRef]

46. Yamada, Y.; Arai, T.; Kato, M.; Kojima, S.; Sakamoto, S.; Komiya, A.; Naya, Y.; Ichikawa, T.; Seki, N. Role of pre-miR-532 (miR-532-5p and miR-532-3p) in regulation of gene expression and molecular pathogenesis in renal cell carcinoma. Am. J. Clin. Exp. Urol. 2019, 7, 11-30.

47. Ross, A.G.; Olveda, R.M.; Chy, D.; Olveda, D.U.; Li, Y.; Harn, D.A.; Gray, D.J.; McManus, D.P.; Tallo, V.; Chau, T.N.; et al. Can mass drug administration lead to the sustainable control of schistosomiasis? J. Infect. Dis. 2015, 211, 283-289. [CrossRef]

48. Weerakoon, K.G.; Gordon, C.A.; Williams, G.M.; Cai, P.; Gobert, G.N.; Olveda, R.M.; Ross, A.G.; Olveda, D.U.; McManus, D.P. Co-parasitism of intestinal protozoa and Schistosoma japonicum in a rural community in the Philippines. Infect. Dis. Poverty 2018, 7, 121. [CrossRef]

49. Barsoum, R.S.; Esmat, G.; El-Baz, T. Human schistosomiasis: Clinical perspective: Review. J. Adv. Res. 2013, 4, 433-444. [CrossRef]

50. Sung, S.; Kim, J.; Jung, Y. Liver-derived exosomes and their implications in liver pathobiology. Int. J. Mol. Sci. 2018, 19, 3715. [CrossRef]

51. Kostallari, E.; Hirsova, P.; Prasnicka, A.; Verma, V.K.; Yaqoob, U.; Wongjarupong, N.; Roberts, L.R.; Shah, V.H. Hepatic stellate cell-derived platelet-derived growth factor receptor-alpha-enriched extracellular vesicles promote liver fibrosis in mice through SHP2. Hepatology 2018, 68, 333-348. [CrossRef] [PubMed]

52. Schwarzenbach, H. Methods for quantification and characterization of microRNAs in cell-free plasma/serum, normal exosomes and tumor-derived exosomes. Transl. Cancer Res. 2017, S253-S263. [CrossRef]

53. Cho, Y.E.; Im, E.J.; Moon, P.G.; Mezey, E.; Song, B.J.; Baek, M.C. Increased liver-specific proteins in circulating extracellular vesicles as potential biomarkers for drug- and alcohol-induced liver injury. PLoS ONE 2017, 12, e0172463. [CrossRef] [PubMed]

54. Chen, L.; Brigstock, D.R. Integrins and heparan sulfate proteoglycans on hepatic stellate cells (HSC) are novel receptors for HSC-derived exosomes. FEBS Lett. 2016, 590, 4263-4274. [CrossRef] [PubMed]

55. Olveda, D.U.; Inobaya, M.T.; McManus, D.P.; Olveda, R.M.; Vinluan, M.L.; Ng, S.K.; Harn, D.A.; Li, Y.; Guevarra, J.R.; Lam, A.K.; et al. Biennial versus annual treatment for schistosomiasis and its impact on liver morbidity. Int. J. Infect. Dis. 2017, 54, 145-149. [CrossRef] [PubMed]

56. Cai, P.; Weerakoon, K.G.; Mu, Y.; Olveda, R.M.; Ross, A.G.; Olveda, D.U.; McManus, D.P. Comparison of Kato Katz, antibody-based ELISA and droplet digital PCR diagnosis of schistosomiasis japonica: Lessons learnt from a setting of low infection intensity. PLoS Negl. Trop. Dis. 2019, 13, e0007228. [CrossRef]

57. Richter, J.; Hatz, C.; Campagne, G.; Bergquist, N.R.; Jenkins, J.M. UNDP/World Bank/WHO Special Programme for Research and Training in Tropical Diseases; Ultrasound in schistosomiasis: A practical guide to the standard use of ultrasonography for assessment of schistosomiasis-related morbidity: Second international workshop, 22-26 October 1996, Niamey, Niger; World Health Organization: Geneva, Switzerland, 1996. 
58. Mu, Y.; Cai, P.; Olveda, R.M.; Ross, A.G.; Olveda, D.U.; McManus, D.P. Parasite-derived circulating microRNAs as biomarkers for the detection of human Schistosoma japonicum infection. Parasitology 2019, 1-8. [CrossRef]

59. Andersen, C.L.; Jensen, J.L.; Orntoft, T.F. Normalization of real-time quantitative reverse transcription-PCR data: A model-based variance estimation approach to identify genes suited for normalization, applied to bladder and colon cancer data sets. Cancer Res. 2004, 64, 5245-5250. [CrossRef]

60. Vandesompele, J.; De Preter, K.; Pattyn, F.; Poppe, B.; Van Roy, N.; De Paepe, A.; Speleman, F. Accurate normalization of real-time quantitative RT-PCR data by geometric averaging of multiple internal control genes. Genome Biol. 2002, 3, RESEARCH0034. [CrossRef]

61. Pfaffl, M.W.; Tichopad, A.; Prgomet, C.; Neuvians, T.P. Determination of stable housekeeping genes, differentially regulated target genes and sample integrity: BestKeeper-Excel-based tool using pair-wise correlations. Biotechnol. Lett. 2004, 26, 509-515. [CrossRef]

62. Silver, N.; Best, S.; Jiang, J.; Thein, S.L. Selection of housekeeping genes for gene expression studies in human reticulocytes using real-time PCR. BMC Mol. Biol. 2006, 7, 33. [CrossRef] [PubMed]

63. Xie, F.; Xiao, P.; Chen, D.; Xu, L.; Zhang, B. miRDeepFinder: A miRNA analysis tool for deep sequencing of plant small RNAs. Plant Mol. Biol. 2012, 80, 75-84. [CrossRef] [PubMed]

(C) 2020 by the authors. Licensee MDPI, Basel, Switzerland. This article is an open access article distributed under the terms and conditions of the Creative Commons Attribution (CC BY) license (http://creativecommons.org/licenses/by/4.0/). 